\title{
Aportes de la administración moderna a la gerencia educativa
}

\author{
Víctor Julio Baltodano Zúniga \\ Director Académico, Campus Nicoya, Universidad Nacional \\ Nicoya, Costa Rica \\ Ana Rita Badilla Alvarado ${ }^{2}$ \\ Escuela Presbítero José Daniel Carmona Briceño \\ Nandayure, Guanacaste, Costa Rica
}

Recibido 23 de marzo de 2009 • Aceptado 29 de abril de 2009

\begin{abstract}
Resumen. En la actualidad, el gerente educativo, o director, dispone de una serie de herramientas de la administración moderna que permiten guiar la institución educativa hacia la calidad. Para ello, debe cumplir con una serie de funciones que se enmarcan dentro del proceso administrativo, tales como: planear, organizar, dirigir y controlar las actividades del centro a educativo. Este escrito tiene como objetivo dar a conocer algunos de estos aportes y la manera en que el gerente educativo puede utilizarlos para mejorar la institución educativa. La administración moderna pone a disposición de éste diferentes concepciones, que ayudan a visualizar la forma en que se debe gerenciar la institución.

La principal conclusión que se extrae es que no existe un cuerpo teórico único que garantice el éxito a un gerente educativo, sino que se debe utilizar lo mejor

de cada una de las concepciones que aporta la administración moderna, y adaptarlas, de acuerdo con las circunstancias.
\end{abstract}

Palabras clave. Administración moderna, gerencia educativa, educación.

\footnotetext{
${ }^{1}$ Magister en Administración de Empresas por el Instituto Tecnológico de Costa Rica. Licenciado en Economía por la Universidad Nacional. Ha tenido experiencia relevante en la administración universitaria. Ha sido Director y Subdirector de la Sede Regional Chorotega de la Universidad Nacional. Ha sido profesor de posgrado de la Universidad Latina de Costa Rica y Universidad de Costa Rica. Actualmente, es docente de la Sede de Guanacaste de la UCR y Director Académico del Campus de Nicoya de la UNA. Tiene más de 15 publicaciones en revistas nacionales e internacionales.

Correo electrónico: vbaltoda@una.ac.cr

${ }^{2}$ Magíster en Administración Educativa por la Universidad Libre de Costa Rica y Licenciada en Pedagogía con énfasis en I y II Ciclos por la Universidad Nacional. Tiene una vasta experiencia en educación primaria en escuelas rurales. Actualmente, es docente de la Escuela Presbítero José Daniel Carmona Briceño, de ciudad Carmona, Nandayure. Tiene publicaciones en revistas nacionales.

Correo: aribadal@costarricense.cr
} 
Abstract. Nowadays, the educative manager or principal has a series of tools of modern administration that allow him/her to guide the educational institution towards quality. Thus, he/she must fulfils a series of functions that are framed within the administrative process, such as planning, organizing, directing and controlling activities in the educational institution. This article's main objective is to present some of these contributions and the way in which the principal can use them to improve the educational institution. The modern administration puts at the disposal different conceptions that help to visualize the way in which the institution must be managed.

The main conclusion that is extracted is that a unique theoretical framework does not exist that can ensure the success of a principal, instead, there must be a use of the best aspects that each of the conceptions that the modern administration have in order to adapt them, according to the circumstances.

Key words. Modern Administration, Educational management, Education.

\section{Introducción}

La práctica gerencial educativa está íntimamente ligada con la forma en que el gerente o director dirige e involucra a las personas que tiene a su alrededor. Por supuesto, todas estas prácticas están dentro de lo que se conoce como el proceso administrativo: planear, organizar, integrar al personal, es decir, todo lo relacionado con la contratación y la administración de los recursos humanos, o sea, dirigir y controlar las actividades de la institución educativa.

La administración moderna aporta a la gerencia educativa todos los conceptos del proceso administrativo, a saber: planeación, organización, dirección, integración de personal y control, pero, también, conceptos relevantes que se integran en el proceso administrativo. La planeación es el primer paso dentro de este proceso. Chiavenato (2002) la define como:

lo que pretende realizar la organización en el futuro y cómo debe realizarlo. Por esta razón, la planeación es la primera función administrativa, y se encarga de definir los objetivos para el futuro desempeño organizacional y decide sobre los recursos y tareas necesarios para alcanzarlos de manera adecuada. (p. 17)

La dirección de la institución educativa, por medio de la planeación, es la encargada de hacer que la misión y la visión se cumplan; establece los objetivos estratégicos y las acciones para lograrlos y, además, toma las decisiones que guiarán el rumbo de mediano y largo plazo de la institución educativa. Para esto, las habilidades de tipo conceptual y de diseño son fundamentales. Una habilidad de tipo conceptual, de acuerdo con Villalta (2000, p.16) "es la facultad de reconocer los elementos importantes en una situación y comprender la relación entre ellos, ver la imagen de conjunto", mientras que una habilidad de diseño "es la aptitud para solucionar problemas de forma que la empresa se beneficie. El arreglo ha de ser factible de acuerdo con la realidad enfrentada" (p. 17). Es importante que en la fase de planeación se informe a los docentes y al personal administrativo sobre lo que se está diseñando, y se busquen estrategias para involucrarlos en la formulación del Plan Estratégico Institucional.

En cuanto a la organización como función administrativa, el gerente educativo o director, ordena y distribuye el trabajo, es la autoridad y decide los recursos por asignar. En este campo, debe quedar claro el rol que cada uno de los integrantes de la organización tendrá dentro de ella, y el ligamen que pueda existir con los demás integrantes de los diferentes equipos de trabajo que existan. Debe existir concordancia con lo establecido en los planes institucionales, ya que no debe haber divorcio entre estas dos funciones. 
A la integración del personal, gurús como Chiavenato prefieren llamarla integración del talento humano, pero la incluyen dentro de la organización como proceso administrativo, y se considera que es otra función a la que la gerencia educativa debe prestar especial atención. Es aquí donde se empieza el éxito o el fracaso del desempeño de la gerencia educativa, ya que si no se identifica, se selecciona y se contrata al colaborador idóneo, la organización podría perder efectividad en la consecución de sus metas y objetivos. Pero, además, implica una clara política de ascensos, de capacitación, de remuneración y del desarrollo de los colaboradores, todas ellas áreas muy sensibles en la administración en tiempos en los que impera la búsqueda de la calidad en la educación. Lamentablemente, en las instituciones de educación costarricense, sobre todo, estatales, el director o gerente educativo, no puede seleccionar a su personal y debe trabajar con perfiles que son impuestos por otros mecanismos de selección, los cuales no garantizan la idoneidad.

La dirección, como función de la administración educativa, es, también, de vital importancia, y trata de la forma en que influimos sobre las personas para que éstas aporten su esfuerzo en aras de conseguir las metas organizacionales y grupales. Es relevante apuntar que el gerente educativo, o director, debe tener claros ciertos conceptos tales como: liderazgo, motivación y comunicación, entre otros, que caen dentro de su ámbito.

De acuerdo con Koontz y Weihrick (2004), el liderazgo es el "arte o el proceso de influir personas para que se esfuercen voluntaria y entusiastamente para lograr las metas del grupo” (p. 532). Es decir, liderazgo significa convencer, persuadir y orientar más que imponer o amenazar.

En cuanto al concepto de motivación, Chiavenato (2002) lo plantea de la siguiente forma: "es el deseo de dedicar gran esfuerzo a los objetivos organizacionales, condicionado por la capacidad del esfuerzo para satisfacer alguna necesidad individual" (p. 632), de aquí que conocer las necesidades de los docentes y los administrativos de la institución es de vital importancia para poder satisfacer sus necesidades.

Dentro del campo de la comunicación Stoner (1997) la define como "el proceso mediante el cual las personas tratan de compartir significados por medio de la transmisión de mensajes simbólicos" (p. 575). Su importancia estriba en que es por medio de ella que se puede producir la coordinación, establecer y difundir los objetivos de la institución educativa, desarrollar los planes institucionales, crear el clima de participación, verificar el desempeño de los docentes y los administrativos, entre otros muchos aspectos.

Por último, dentro del ámbito gerencial educativo, el control también es importante, a causa de que solamente de esta forma se puede saber si se está cumpliendo con las metas establecidas. El control tiene su fundamento en la posibilidad de medir y corregir el desempeño individual y organizacional para asegurar que las actuaciones se ajusten a lo planeado (Villalta, 2000)

En la búsqueda de ser mejor, el gerente educativo o director de una institución educativa, cuenta con una serie de herramientas conceptuales que apoyan su accionar En este artículo se retoman, someramente, algunas de ellas y se concluye con una serie de reflexiones para la gerencia educativa en los nuevos ambientes de la modernidad.

\section{El comportamiento organizacional como disciplina para entender las instituciones educativas}

El Comportamiento Organizacional (C.O) es una disciplina importante para la comprensión de las organizaciones. Ésta se define como "el campo de estudio que investiga las repercusiones que los individuos, los grupos y la estructura producen en el comportamiento de las organizaciones, con 
el propósito de aplicar estos conocimientos para mejorar la eficacia de una organización" (Robbins, 2004, p. 8). Esta disciplina, en última instancia, busca estudiar el impacto del comportamiento de las personas sobre el rendimiento de la organización, la satisfacción laboral y la rotación del personal. Esta comprensión es fundamental para una institución educativa, dado que ésta es la formadora de los niños y los jóvenes del país. Esta tarea debe hacerse con calidad, y parte de los docentes y los administrativos y su forma de actuar en la institución.

Otro aspecto relevante por tomar en cuenta, es que todas las organizaciones tienen una estructura la cual se define como un "esquema formal de relaciones, comunicaciones, procesos de decisión, procedimientos y sistemas dentro de un conjunto de personas, unidades, factores materiales y funciones con vistas a la consecución de objetivos" (Zerilli, citado por Gómez, 2007, p. 5). En el caso del sistema educativo, éste es dirigido desde el Ministerio de Educación Pública. En esta estructura, de acuerdo con Gómez (2007), se pueden distinguir cuatro niveles:

1. En este nivel están los dirigentes, está integrado por los funcionarios de altos puestos del sistema escolar, los cuales tienen a su cargo la estructuración general de la enseñanza, así como la elaboración de los planes generales de acción.

2. En este el nivel se encuentran los inspectores o supervisores, éstos se distribuyen en zonas o sectores regionales, y les corresponde orientar y controlar los procedimientos y eficacia de la acción.

3. Este nivel está integrado por los directores de los centros educativos, quienes ejercen la autoridad sobre los docentes a su cargo, y tienen como funciones, velar por la labor organizativa y parte de la didáctica de la escuela, así como vigilar el rendimiento de los educandos y sus colaboradores, y mantener las relaciones con la comunidad.

4. En este nivel están los docentes que ejercen las tareas en relación directa con los alumnos, ejercen una función directamente didáctica, combinada con una parte organizativa, en cuanto a la adaptación de los objetivos de la enseñanza mediante los programas y los métodos que se materializan en el aula, deben velar por la calidad académica de los alumnos.

Sin embargo, es necesario rescatar que la teoría administrativa, dentro de la cual se encuentra inmerso el campo del comportamiento organizacional, ofrece a los gerentes educativos, varias formas estructurales para organizar la institución y organizar el trabajo. Es necesario que escojan aquella que se adapte mejor a las circunstancias y a las características de la institución.

Otro de los aspectos fundamentales en el análisis del comportamiento de las organizaciones son sus valores, las motivaciones y las preferencias de las personas, el clima, la cultura y el diseño organizacional. El estudio de todos estos aspectos permite comprender, de forma integral, a las organizaciones y, en este caso, a las instituciones educativas. Esta comprensión lleva al éxito de la gerencia educativa.

En el caso del MEP, es necesario un cambio de cultura, el cual permita alcanzar una mejor calidad en la educación. Además, debe diseñarse un nuevo sistema de incentivos que permita girar hacia un sistema de mérito, es decir, que haya una política clara de ascensos o de mejoramiento en el escalafón docente, en el que se valore la búsqueda de la calidad, la capacitación continua, la producción intelectual, el desempeño del docente en el aula, el impacto de la institución en la comunidad, la satisfacción de los padres de familia, entre otros elementos. El gerente educativo, o director, debe tener su cuota en el diseño de este sistema, de modo que impacte no sólo la selección del colaborador, sino, también, su desarrollo y la consecución de los objetivos institucionales. 
La comprensión de los valores, las normas, las motivaciones, entre otros elementos, facilitan la planeación estratégica en una organización, y la institución educativa no es la excepción.

\section{La planeación estratégica como fundamento para alcanzar la calidad}

Otro aporte de la administración moderna a la gerencia educativa es la planeación estratégica. Esta teoría se ha aplicado, en mayor medida, al mundo empresarial y ha tenido éxito. Sin embargo, las instituciones públicas también la han aplicado para mejorar sus servicios, y en las instituciones educativas, debe utilizarse para un mejoramiento integral.

Esta planeación debe ser estratégica, de tal forma que se alcancen objetivos de gran impacto, que conduzcan a un verdadero crecimiento en la calidad de la institución.

De acuerdo con Chiavenato (2002), planeación "es la función administrativa que define objetivos y decide sobre los recursos y tareas necesarios para alcanzarlos adecuadamente" (p. 214). Esta planeación se manifiesta en las directrices que sirven de guía para alcanzar las metas en la institución educativa.

La planeación estratégica puede definirse como el proceso amplio de adaptación organizacional que implica aprobación, toma de decisiones y evaluación; busca responder preguntas básicas como por ejemplo: por qué existe la organización, qué hace y cómo lo hace. El resultado del proceso es un plan que sirve para guiar la acción institucional en un plazo de tres a cinco años. Parafraseando a Jofré (1999), la planificación estratégica puede definirse como el proceso por medio del cual una institución educativa define su propósito, con una visión de largo plazo y, selecciona alternativas existentes en un contexto o comunidad determinado, las cuales se expresan, fundamentalmente, por medio de objetivos estratégicos y estrategias de implementación.

De acuerdo con estas definiciones, puede concluirse que, para cualquier institución educativa, es necesario tener claro el rumbo por el cual va a transitar. Esto significa que se debe tener una visión de largo plazo, y no trabajar sólo a corto plazo y de forma rutinaria. De ahí que la formulación de objetivos estratégicos, y de una estrategia para alcanzarlos, ayuda a que una institución mejore continuamente.

La planeación estratégica presenta cinco características contextualizadas, de acuerdo con Chiavenato (2002):

- La planeación estratégica se relaciona con la adaptación de la organización a un ambiente variable. En el caso de una institución educativa, debe llevarse a cabo un análisis FODA (fortalezas, oportunidades, debilidades y amenazas) que permita ver las debilidades y las oportunidades, así como las amenazas y las fortalezas. Se debe incluir tanto el contexto internacional como el nacional y hasta el ámbito de la comunidad. Asimismo, es necesario tener claro el diagnóstico interno que ayude a potenciar las fortalezas y atacar las debilidades.

- La planeación estratégica se orienta hacia el futuro. Permite proponer metas estratégicas que deberán ser alcanzadas en un período establecido de antemano, y que ubiquen a la institución educativa en un escenario mejor al actual.

- La planeación estratégica es amplia. Incluye a todos los niveles de la institución educativa y no sólo a un departamento, un nivel (grado) o una sección.

- La planeación estratégica es un proceso de construcción de consenso. Se debe involucrar a la mayor cantidad de personas, no tanto físicas, sino de ideas o representantes de esas ideas. 
- La planeación estratégica es una forma de aprendizaje organizacional. Es un ejercicio que obliga a estar pensando y repensando a la institución, con el fin de hacer mejor las cosas y corregir las irregularidades.

Tres son las preguntas fundamentales que se debe hacer a la hora de realizar este tipo de planeación: 1) ¿Dónde estamos? Sería el diagnóstico de la institución educativa incluido su entorno externo. Es la realidad institucional y del sector educativo. 2) ¿Hacia dónde vamos? Es el escenario futuro; conjunto de metas estratégicas que se quieren alcanzar para mejorar la institución. 3) ¿Cómo llegaremos ahí? Consiste en valorar los recursos con los que se cuenta, las estrategias que se van a impulsar, las políticas, las normas, los procedimientos que van a permitir alcanzar lo planeado.

El éxito de una institución educativa está en tener claro cuál es su misión, establecer, de manera precisa, una visión, formular objetivos o metas estratégicas que verdaderamente vayan a ubicar a la institución en una mejor posición, y en seleccionar estrategias que permita alcanzar esos propósitos. Es conveniente que la institución se proponga una o dos metas estratégicas, que sean de verdadero impacto, y ligar a ellas todos los demás objetivos. No se debe perder el rumbo proponiendo metas rutinarias o abstractas.

De acuerdo con Chiavenato (2002) y adaptando sus ideas a una institución educativa, son seis pasos con los que cuenta, habitualmente, la planeación:

1. Definir los objetivos. Consiste en establecer los objetivos estratégicos que se pretende alcanzar. Los objetivos de la institución educativa deben orientar los principales planes (por ejemplo: plan de capacitación, plan de mejoramiento de la infraestructura, etc.) y servir de base a los objetivos departamentales y a los planes de cada grupo o nivel.

2. Verificar la situación actual frente a los objetivos. Se debe evaluar la situación actual en contraposición a los objetivos deseados, verificar dónde se está y qué se debe hacer. Para esto, un diagnóstico institucional es fundamental en el que se establezcan debilidades, fortalezas, oportunidades y amenazas que se ciñen para la consecución de la calidad de la educación.

3. Desarrollar premisas sobre las condiciones futuras. Las premisas constituyen los ambientes esperados de los planes de operación de la institución educativa. Se trata de generar escenarios alternativos para los estados futuros de las acciones, analizando lo que puede ayudar o perjudicar el avance hacia los objetivos. La previsión es un aspecto importante en el desarrollo de premisas y está relacionada con supuestos acerca del futuro.

4. Analizar las alternativas de acción. Se trata de relacionar y evaluar las acciones que se deben emprender, escoger una de ellas para perseguir uno o más objetivos, y elaborar un plan para alcanzarlos.

5. Elegir un curso de acción entre las diversas alternativas. Se trata de una toma de decisión en que se elige una alternativa y se abandonan las demás. La alternativa seleccionada se transforma en un plan para alcanzar los objetivos que la institución considera fundamentales.

6. Implementar el plan y evaluar los resultados. Hacer lo que el plan determina y evaluar, con cuidado, los resultados para garantizar la consecución de los objetivos, seguir lo que fue planeado y emprender las acciones correctivas a medida que sean necesarias.

Una vez comprendidos estos pasos, el gerente educativo debe integrar al personal para que se comprometan con el rumbo de la institución. Solamente con el compromiso decidido de los 
integrantes es que se asegura el éxito. La administración moderna ha aportado otro concepto fundamental que permite que esa planeación estratégica no se quede sólo en el papel, sino que se materialice en las acciones elaboradas; ese concepto es el empowerment.

\section{Empoderamiento: una nueva visión para la integración de personal}

Una definición aceptable de empowerment aduce que "es un proceso que pretende la construcción, desarrollo e incremento del poder de la organización, a través de la cooperación, participación y trabajo conjunto" (Jofré, 1999, p. 17). En otras palabras, significa facultar a los colaboradores, es decir, liberar el conocimiento y la energía de los docentes y administrativos para que compartan información, y tomen decisiones eficaces en equipo. El propósito último, es mejorar, continuamente, la escuela o el colegio al que pertenecen.

Sin embargo, el término que mejor se ajusta a esta forma de trabajo es el de organización inteligente; no obstante, el más utilizado es el de empoderamiento. La organización inteligente es aquella basada en el conocimiento y en la habilidad para aprender; es, precisamente, esta habilidad para aprender de forma más rápida, hecho que podría mantener la pertinencia de la institución educativa, y hacer la diferencia entre el éxito y el fracaso. Para Jofré (1999):

el empowerment se basa en dos columnas: un fuerte cambio en el pensamiento directivo y la creación de un clima que permita un alto grado de compromiso del empleado con su organización. Tratar de construir una organización inteligente basada en la desconfianza y el uso exclusivo de la autoridad formal es un error que no puede subsanarse con prácticas administrativas, aun siendo las que normalmente se aplican en este tipo de enfoques. (p. 26)

Lo anterior implica un reto para la gerencia educativa, dado que el cambio empieza desde arriba, sin olvidar las bases, e impacta la selección y la capacitación del recurso humano que tiene la institución educativa. Por tanto, la gerencia educativa debe realizar un esfuerzo por construir una visión compartida con todos los componentes de la institución; además, crear un alto grado de compromiso del colaborador con ella; asimismo; con sus actuaciones mantener una relación de confianza que involucre a los padres, a los administrativos y a los docentes; incentivar la toma de decisiones por parte de estos últimos y crear un sistema de comunicación en doble vía. Para ello, se podrían implementar los equipos autodirigidos, que se asemejan a células, que reaccionan de manera inteligente a las necesidades de los estudiantes y del entorno. Estos equipos deben tener la libertad de organizarse, y dirigir su trabajo, de acuerdo con las metas que se han fijado en la institución. Son responsables de los resultados y de la evaluación de su trabajo, es decir, se autocontrolan y autorregulan.

El gran reto para la gerencia educativa, o director, que reconoce las ventajas de una organización basada en el aprendizaje constante, consiste en liderar el cambio hacia una cultura organizacional, que adopte y valore el compromiso con el mejoramiento continuo, en la que cada participante se apropie de una visión y una misión compartidas por todos. Este proceso demanda mucho entusiasmo, claridad en los objetivos y metas tanto de corto como de más largo plazo, lo que se puede calificar como parte de una estrategia dual, que atiende las actividades diarias dentro de una agenda, adecuadamente, elaborada y que aborda los procesos de planificación para el largo plazo. Este proceso tiende a ser muy lento, requiere colaboradores con alto grado de compromiso, que permita una estructura muy flexible, ausente de tanto control sobre la gente, y promueva la 
creación de espacios para el estímulo de la creatividad y la innovación, dos elementos esenciales para lograr una ventaja sostenida en las escuelas, y en el sistema educativo, en general.

El camino hacia el crecimiento institucional, basado en la participación efectiva de los colaboradores, va más allá de la creatividad por sí sola; este proceso de participación debe ser planeado, de forma que no se convierta en una frustración, por parte de quienes creen que el solo hecho de estimular la creatividad es suficiente.

Levit (2002) advierte los peligros de una mala canalización de la creatividad, en este caso empresarial:

el problema con la avalancha de consejos (...) acerca de la necesidad de volverse más activamente creativas es el hecho de que sus defensores, en esencia, han fracasado en distinguir entre el proceso relativamente fácil de ser creativos en lo abstracto y el infinitamente más complicado de innovar en lo concreto. (p. 16)

De todas formas, creatividad e innovación, son esfuerzos que se deben realizar en las instituciones educativas costarricenses. Ahí tiene el director, o gerente educativo, una ardua tarea por impulsar.

Más aún, existen muchas y buenas ideas implementadas en las instituciones educativas tanto costarricenses como extranjeras. Estas experiencias innovadoras se pueden utilizar para mejorar el accionar de las instituciones educativas. Para ello, se puede utilizar otra herramienta que la administración moderna ha creado: el benchmarking.

\section{Benchmarking para la calidad en educación}

Comúnmente utilizado en el mundo empresarial el benchmarking puede ser adaptado a la educación. Consiste en captar las mejores ideas, prácticas, procedimientos, estrategias y técnicas que utilizan otras empresas, preferiblemente líderes, y que han sido exitosas; implica compararse con esas empresas, instituciones o unidades de negocios e implantar, en nuestra organización, las que mejor se adapten. Jofré (1999) apunta que "Benchmarking significa punto de referencia. Se trata justamente de eso, de buscar puntos de referencia exitosos en el entorno para compararlos con los nuestros y, si son útiles para nuestra organización, adaptarlos y si es posible mejorarlos" (p. 196). En la institución educativa costarricense, se puede contextualizar para utilizar las mejores prácticas de otros países, inclusive instituciones educativas nacionales, que se han destacado por su desempeño.

En cuanto a las ventajas del benchmarking, de acuerdo con Jofré (1999) se señalan las siguientes:

1. Permite introducir mejoras con bajo costo y poco riesgo. Es cuestión de buscar las mejores ideas y prácticas exitosas en el entorno y compararlas con las de la institución educativa.

2. Disminuye el tiempo para producir cambios efectivos. Otros enfoques, normalmente necesitan más tiempo para producir los cambios y lograr los resultados propuestos.

3. Dirige la institución educativa hacia grandes desafíos. Se enfoca hacia desafíos de excelencia, de alto nivel. Es excelente para fijar objetivos en una institución de educación.

4. Logra un mejoramiento continuo de la escuela o institución educativa. 
Y lo explica de la siguiente forma:

El benchmarking es un medio que tiene la organización para estar captando y adaptando las mejores prácticas del entorno de manera permanente, dado los rápidos cambios que se dan en el mismo. Esto permite el mejoramiento continuo en el desempeño de la organización. (p. 200)

En el mundo de los negocios, el benchmarking es muy utilizado y en las instituciones educativas no debería ser la excepción. Desde hace más de una década, en Business Week (citado por Baltodano, Arguedas y Picón, 2004) se lee:

Ejércitos de ejecutivos del mundo entero quieren visitar compañías estadounidenses de buen desempeño, en busca de ideas y consejos para aplicar en sus propias empresas. (...) Los peregrinos corporativos de hoy, azotados por el flagelo de la globalización, la reestructuración (downsizing) y los cambios tecnológicos, están buscando soluciones y refugio en Motorota, Microsoft y en Springfield ReManufacturing. (p. 83)

Esta actitud de aprender que se da en los negocios debería darse también en la educación. No es posible que, muchas veces, los mismos educadores tengan actitudes mezquinas y no quieran compartir con los colegas las buenas prácticas.

Lo importante del benchmarking es la actitud siempre alerta que debe tener la gerencia educativa, con respecto al entorno. Debe haber un monitoreo continuo de todos los elementos del entorno que puedan afectar, positiva o negativamente, la institución educativa.

Además del benchmarking, la administración moderna también ha aportado otra teoría de gran relevancia para la educación: la calidad total. En boga en los años ochenta, revolucionó el concepto de calidad en las empresas.

\section{Calidad total para la educación}

La calidad total es otro concepto que ha aportado la administración moderna y que puede ser utilizado por la gerencia educativa. Deming (citado por Jofré, 1999) señala que "la calidad significa ofrecer a bajo costo productos o servicios confiables que satisfagan a los clientes, creando un compromiso con la innovación y mejora continua en la empresa" (p. 120). La calidad debe ser permanente y, por otro lado, debe ser un imperativo que refuerce una ventaja competitiva.

La noción de calidad como perfección recurre a la idea de consistencia o ausencia de defectos. Se trata de asegurar que las tareas se realizan de manera correcta desde el principio, asociándose, intrínsecamente, con la idea de una cultura de calidad, lo que implica la responsabilidad de todos los actores de la organización. De acuerdo con Muller y Funnell (citados por Baltodano, 2005) la noción de calidad como transformación "apela al cambio cualitativo o valor agregado que ocurre por lo menos en el educando, en términos de conocimientos, destrezas y habilidades" (p. 18). Su medición no es sencilla, ya que implica definir qué se entiende como valor y una rigurosa evaluación de entrada y salida; por lo demás, la transformación de personas es afectada por múltiples factores que, muchas veces, escapan al control de la institución.

En la noción de valor por dinero, la calidad se asocia con "retorno o rentabilidad que se logra respecto de la inversión que se realiza. En este enfoque, subyace el concepto de "rendición 
de cuentas" ante quienes sostienen la institución, lo que corresponde al término en inglés de "accountability" (Cave, Kogan, Smith, 1990, citados por Baltodano, 2005, p.18).

La calidad es mayor si el mismo resultado se logra con menos recursos o mejor resultado al mismo costo. La noción de adecuación o aptitud para el logro de los propósitos, asocia la calidad con la satisfacción o cumplimiento de los requerimientos y necesidades o deseos de los usuarios.

La calidad de la educación se hace realidad en los aprendizajes cualitativamente relevantes. La calidad no está en lo que se enseña, sino en lo que se aprende, por lo que, en la práctica, está cada vez más centrada en el propio sujeto educativo. La calidad tiene que ver con: el currículo, los métodos de enseñanza, los medios, la formación de los profesores, el ambiente pedagógico y la investigación educativa.

En cuanto al Sistema ISO (International Standard Organization) concibe la calidad como la totalidad de partes y características de un producto, que influyen en la aptitud para satisfacer las necesidades de los clientes. Por tanto, calidad es cumplir con las especificaciones que hace el cliente de determinado producto o servicio, en este caso, el educativo. De acuerdo con las normas ISO, el aseguramiento de la calidad son todas las acciones planeadas para brindar la confianza de que un producto o servicio va a satisfacer determinados requerimientos de calidad en forma adecuada. El gerente educativo debe buscar la forma de asegurar la calidad de la educación. Por ejemplo, en la educación superior existe el Sistema Nacional de Acreditación de la Educación Superior (SINAES), el cual está intentando crear un sistema sólido para asegurar la calidad de la educación superior. En la educación primaria, falta crear un sistema que asegure la calidad; y en el nivel de secundaria, los exámenes de bachillerato no han cumplido con las expectativas.

Ríos (2009) se refiere al caso del Perú y anota:

En nuestro país hasta donde nos da la experiencia, podemos decir que a nivel de política de Estado la Calidad total no se ha insertado en la estructura como lo es por ejemplo, Japón, pues no ha pasado de ser solo eslóganes, buenos propósitos y algunas acciones orientadas a una cultura de calidad como lo es el caso de la salud y educación. Lo que sí es bueno entender es que la calidad es una fórmula de sobrevivencia y para eso se requiere ir agregando capas y capas de calidad que irá apuntando hacia la calidad total (TQM) que será la meta de toda institución educativa moderna. Entonces de lo que se trata es de desarrollar calidad desde la cultura misma de la institución pasando por criterios, normas y finalmente el TQM. (s. p.)

Lo anterior no se aleja mucho de la realidad costarricense. Sin embargo, lo más importante para una institución educativa, en cuanto a las normas ISO, es la imagen que pueda proyectarse hacia el entorno de un esfuerzo continuo de mejoramiento de la calidad. Asimismo, las normas ISO, generalmente, mejoran el desempeño y la comunicación del personal; obliga a la documentación de todos los procesos, y permite que el conocimiento permanezca en la institución, y no sólo en la mente de los profesores, maestros y directores experimentados.

Debe quedar claro que el gerente educativo, o director, debe tomar de los aportes de la administración moderna solamente aquellas premisas que permitan un verdadero crecimiento de la institución. Lo anterior, en el sentido de que la administración moderna ayuda a realizar una mejor gestión, pero no es la solución a todos los problemas. Es más, se debe tener cuidado con no mercantilizar la educación, sino buscar la calidad, pero sin perder el humanismo, la solidaridad y los valores del ser costarricense. 


\section{Conclusiones}

1. Todo gerente educativo debe tener claro que existe un proceso administrativo que empieza con la planeación. Esta planeación debe ser estratégica, para que sea relevante y permita alcanzar las metas que persigue la institución educativa. Se debe tener claro que el norte debe ser la búsqueda de la calidad de la educación.

2. Dentro del proceso administrativo se encuentran cuatro fases más, además de la planeación, a saber: la organización, la dirección, la integración de personal y el control. Estas cinco fases están interrelacionadas y conducen a lo que se conoce como las funciones del administrador: planear, organizar, dirigir, integrar el personal y controlar.

3. Constantemente, surgen aportes de la administración moderna que enriquece la forma como se implementa el proceso administrativo. Dentro de estos aportes están la teoría del comportamiento organizacional, el empowerment, el benchmarking y la calidad total, solamente por mencionar las más importantes.

4. Todos esos aportes de la administración moderna pueden ser integrados por el gerente educativo, para mejorar la institución educativa. Por ejemplo, la teoría del comportamiento organizacional, se preocupa por los individuos, los grupos y la estructura organizacional y cómo esa interrelación puede impactar el rendimiento, la satisfacción laboral y la rotación del personal. Por tanto, comprender el marco de valores, los elementos motivacionales, los canales de comunicación y la forma como se lidera son factores fundamentales para el avance de una institución educativa. La clara comprensión de esos factores puede hacer la diferencia entre un buen clima organizacional o uno hostil.

5. La planeación estratégica es otro aporte de la administración moderna que puede ser utilizado por la gerencia educativa. Es necesario tener un rumbo, es decir, una direccionalidad, y un conjunto de metas hacia las cuales se pretende llegar. Los integrantes de la institución educativa, en conjunto con el gerente educativo, deben definir estas metas; de tal forma, que sean estratégicas, que permitan llevar a la institución hacia mejores estándares de calidad. Esta claridad de las metas permite elaborar los planes institucionales con un verdadero valor para la toma de decisiones, y no sólo como instrumentos para justificar acciones de corto plazo. Para que esas metas sean estratégicas se debe estudiar el entorno externo e interno y seleccionar las alternativas viables, para cumplir con la misión y la visión.

6. Otro de los aportes de la administración moderna a la educación es el benchmarking. Captar las mejores ideas, prácticas, procedimientos, estrategias y técnicas del entorno permite tener un referente de instituciones de éxito que se pueden incorporar en el Plan Institucional del establecimiento educativo. Lo importante del benchmarking es la actitud siempre alerta que debe tener la gerencia educativa con respecto al entorno. Debe haber un monitoreo continuo de todos los elementos del entorno que puedan afectar, positiva o negativamente, la institución educativa.

7. La calidad total es otro marco conceptual que la administración moderna ha construido, y que es de factible utilización en la educación. La calidad de la educación se hace realidad en los aprendizajes cualitativamente relevantes. No está en lo que se enseña, sino en lo que se aprende, por lo que, en la práctica, está cada vez más centrada en el propio sujeto educativo. Tiene que ver con el currículo, los métodos de enseñanza, los medios, la formación de los profesores, el ambiente pedagógico y la investigación educativa. 
8. De todo lo anterior, se extrae que no existe un cuerpo teórico único que garantice el éxito a un gerente educativo, sino que se debe utilizar lo mejor de cada una de las concepciones que aporta la administración moderna, y adaptarlas, de acuerdo con las circunstancias.

\section{Referencias bibliográficas}

Baltodano, V. J., Arguedas, C. \& Picón, J. (2004). Enfoques y estilos gerenciales modernos adaptados a ambientes internacionales. Revista Geográfica de América Central, 39, 75-91.

Baltodano, V. J. (2005). La acreditación en la Educación Superior como búsqueda de la calidad y la excelencia. Heredia, Costa Rica: Programa de Publicaciones e Impresiones de la Universidad Nacional.

Chiavenato, I. (2002). Administración en los nuevos tiempos. Bogotá, Colombia: McGraw Hill.

Gómez, R. (2007). Gerencia educativa contemporánea. Capítulo 1 de Tesis Doctoral. Universidad Libre de Costa Rica.

Jofré, A. (1999). Enfoques gerenciales modernos. San José, Costa Rica: Ediciones Delphi.

Koontz, H. \& Weihrick, H. (2004). Administración. Una perspectiva global. (12ª ed.). México: McGraw Hill.

Levit, T. (2002). La creatividad no es suficiente. SUMMA, 101, 14-19.

Ríos, M. (2009). Estilos de gestión para una educación de calidad. Revista Investigando. Recuperado el 11 de junio del 2009, de www.scribd.com/doc/4463923

Robbins, S. (2004). Comportamiento organizacional. (10ª ed.) México: McGraw Hill.

Stoner, J. (1997). Administración. México: Editorial Prentice Hall.

Villalta, G. (2000). Curso superior de administración. San José, Costa Rica: Editorial Sello Latino. 